\title{
Endodontic Management of External Apical Root Resorption: A Case Report
}

\author{
Nikita Sarraf ${ }^{1}$, Akansha Jharwal ${ }^{2}$, Deepak Raisingani ${ }^{3}$, Ashwini B Prasad ${ }^{4}$, Harshit Srivastava $^{5}$, Prachi Mittal $^{6}$
}

\begin{abstract}
Resorption is the combination of either physiological or pathological factors that results in the loss of dentin, cementum, and the alveolar bone of tooth structure due to the action of polynuclear giant cells. The most frequent causative factor for resorption is local factors, notably excessive pressure and inflammatory process. Different types of resorption have been classified depending upon its etiology and site, and different treatment regimens have been proposed based on the type of resorption present. External apical root resorption is usually pathological. The case in this article demonstrates external root resorption managed with nonsurgical root canal therapy with the use intracanal medicament of calcium hydroxide, with 1 month follow-up revealing arrest of resorption. Subsequent follow-up at 6 months shows complete repair of periradicular and furcation area.

Keywords: Calcium hydroxide, External root resorption, Nonsurgical treatment, Pathological, Physiological.

Journal of Mahatma Gandhi University of Medical Sciences \& Technology (2019): 10.5005/jp-journals-10057-0101
\end{abstract}

\section{INTRODUCTION}

Root resorption can be described as a condition causing progressive loss of dentine and cementum resulting because of either a physiologic or a pathologic process. ${ }^{1}$ According to Imfeld, "Resorption can occur either by biological degradation or assimilation of structures or substances produced previously in the body". ${ }^{2}$ According to Fuss, resorption occurs due to damage of tissues either by mechanical or chemical means, which is further stimulated by infection or pressure. ${ }^{3}$

In the affected tooth, external root resorption usually begins in the periodontium of altering either the external or the lateral surfaces of a tooth. Many factors are contributory in root resorption which may appear as separate or joined defects. ${ }^{2}$ An inflammatory resorption can occur due to traumatic injuries, orthodontic tooth movement, tooth whitening agents, pulpal necrosis, and chronic infection of the periodontal structures, which can initiate an inflammatory response within the periodontal ligament.

Minor irritants can lead transient resorption, which can be reversed on eliminating the causative stimulus. ${ }^{4}$ The factors that are commonly associated with external root resorption are cysts, tumors, dental trauma, periapical inflammation, orthodontic therapy, pressure from impacted tooth, reimplantation of tooth, endocrine disturbances.

Prognosis of resorption can be improved by proper diagnosis and treatment planning. For proper diagnosis of the site and extent of the lesion, periapical radiographs are suggested to be taken from different angulations and cone-beam computed tomography $(C B C T)$ for helping in identification.

For healing to take place in the periradicular space, removal and destruction of bacteria are the ultimate treatment goal in the external apical root resorption. Appropriate management strategies should be followed, including removal of source of stimulus, reduced osteoclastic activity, stimulating repair, or a combination of these methods once a proper diagnosis is made. ${ }^{5}$

This article presents case report on management of external apical root resorption by nonsurgical root canal treatment with the use of calcium hydroxide as intracanal medicament.
${ }^{1-6}$ Department of Conservative Dentistry and Endodontics, Mahatma Gandhi Dental College, Mahatma Gandhi University of Medical Sciences and Technology, Jaipur, Rajasthan, India

Corresponding Author: Akansha Jharwal, Department of Conservative Dentistry and Endodontics, Mahatma Gandhi Dental College, Mahatma Gandhi University of Medical Sciences and Technology, Jaipur, Rajasthan, India, Phone: +91 8107826629 , e-mail: aakanshajharwal2093@gmail.com

How to cite this article: Sarraf N, Jharwal A, Raisingani D, et al. Endodontic Management of External Apical Root Resorption: A Case Report. J Mahatma Gandhi Univ Med Sci Tech 2019;4(1):18-20.

Source of support: Nil

Conflict of interest: None

\section{Case Description}

A 20-year-old male patient reported to department of conservative dentistry and endodontics with the chief complaint of pain in right lower back tooth region since past 10 days. The patient gave no history of trauma or orthodontic treatment. The medical history was noncontributory. Clinical examination revealed mesioproximal caries in mandibular second premolar (\#45). Palpation and percussion tests induced discomfort and pain. The periapical radiographic film revealed the presence of shortened, flattened apex with substantial periapical radiolucency at the apex in \#45 with the type IV canal configuration (according to Weine's classification) (Fig. 1). Based on the clinical and radiographic findings, the diagnosis was irreversible pulpitis with external apical inflammatory root resorption in \#45. Conventional root canal treatment combined with calcium hydroxide dressing was recommended as the treatment modality.

Endodontic therapy was initiated in \#45 after local anesthesia administration and isolation with a rubber dam (GDC, Hoshiarpur, Punjab). An access cavity was prepared using Endo Access bur (Dentsply Maillefer, Ballaigues, Switzerland). Working length was performed using 15 no. K file (Dentsply Maillefer, Ballaigues, Switzerland) using apex locator Root ZX mini (J. Morita, USA) 


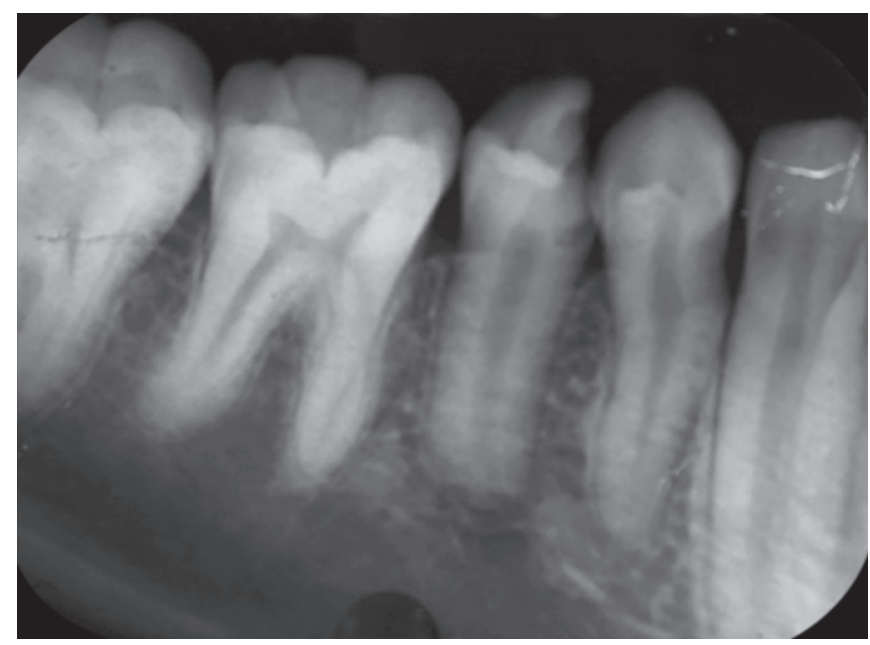

Fig. 1: Preoperative radiograph

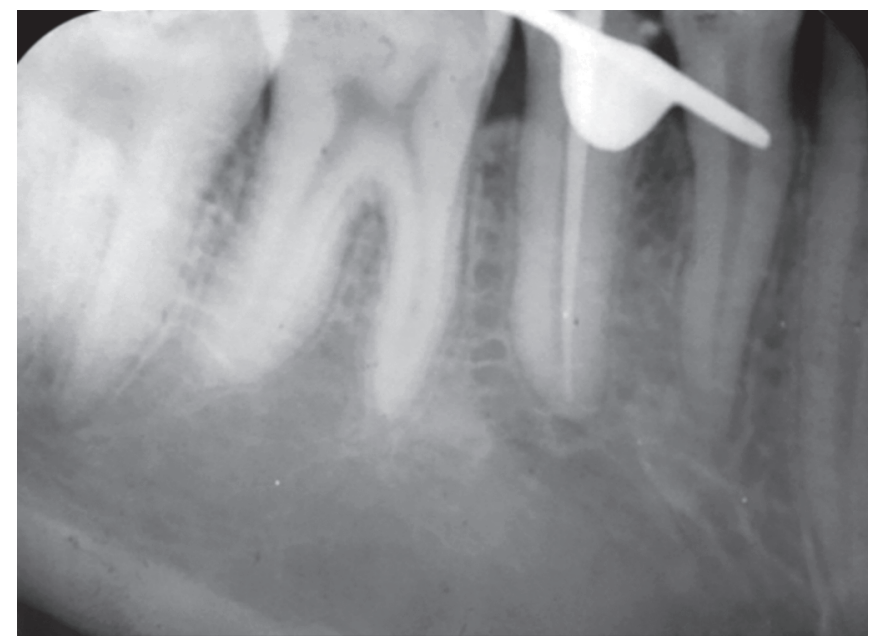

Fig. 3: Master cone radiograph

and was confirmed radiographically (Fig. 2). Chemomechanical preparation was done with rotary protaper (Dentsply Maillefer, Ballaigues, Switzerland) using EDTA (Glyde, Dentsply Maillefer, Ballaigues, Switzerland) up to F2 with crowndown technique and copious irrigation with $5.25 \%$ sodium hypochlorite with intermittent rinse with saline. The canals were dried using sterilized paper points (Dentsply Maillefer, Ballaigues, Switzerland) and filled with calcium hydroxide paste (Metapex, Meta dental corp., New York) as an intracanal medicament, and closed dressing was placed (Cavit G, 3M ESPE, Germany) for 21 days, and the patient was again recalled for change in dressing for a week.

One month later, the patient was recalled. Patient was asymptomatic, and the medicament was removed with 5.25 sodium hypochlorite and saline and using $\mathrm{H}$ file circumferentially, and the root canal was dried using paper point. Radiographically, the tooth revealed good periapical healing and no further shortening and flattening of root apex. After the master cone selection (Fig. 3), the canal was then obturated with lateral compaction technique using F2 Gutta Percha (Dentsply Maillfefer, Ballaigues, Switzerland) and sealer AH plus (Dentsply, De Trey, Germany) (Fig. 4). The tooth received a permanent posterior composite resin core (3M Filtek P60). Post-endodontic rehabilitation was done with PFM crown. At

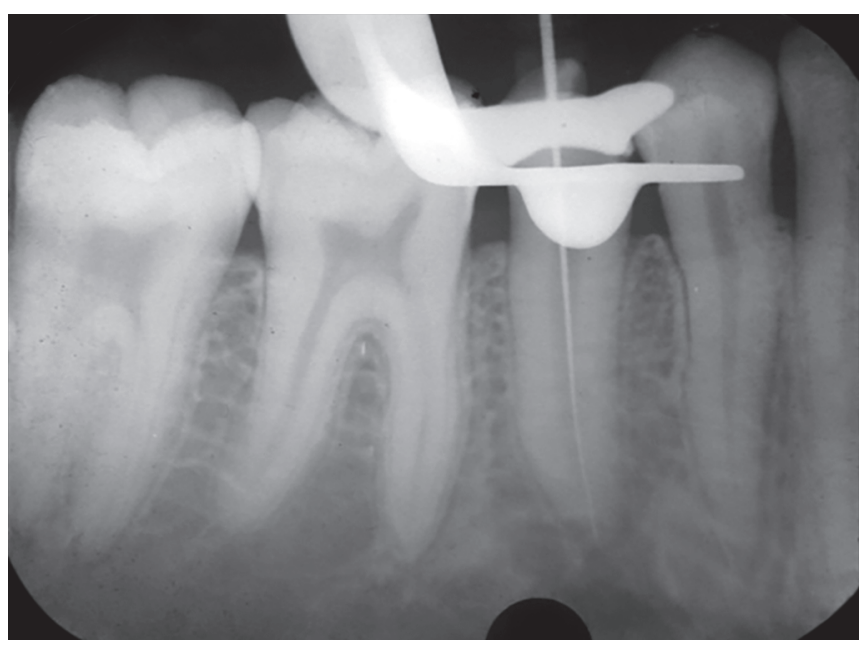

Fig. 2: Working length radiograph

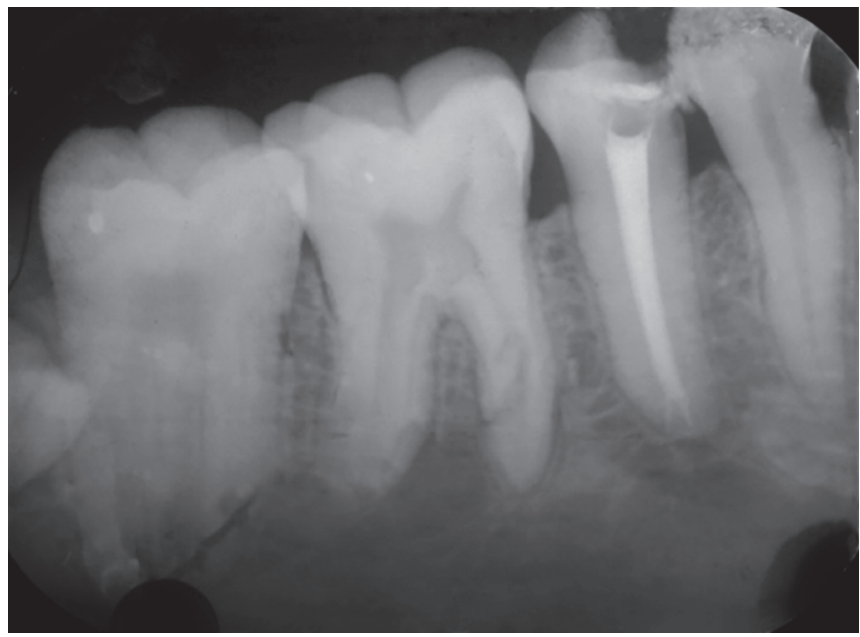

Fig. 4: Obturation radiograph

follow-up visits on the sixth month (Fig. 5), 1 year (Fig. 6), and 2 years (Fig. 7), there was arrest of resorption with no further shortening and flattening of root radiographically, and no changes in the crown root ratio were noted.

\section{Discussion}

Within the root canal system and in the periodontal tissues, inflammation is stimulated by bacteria, bacterial by-products, and tissue breakdown products, which may lead to progressive and aggressive inflammatory resorption of the root. ${ }^{2}$ Sometimes, managing resorptive lesions can be challenging with unknown outcomes. Total loss of root structure can occur through rapidly progressing event of resorption. There are various etiologies of external root resorption, and the case presented in this article had irreversible pulpitis as the major etiology leading to external inflammatory root resorption in the apical region of the root.

External root resorption in single tooth seems to be a relatively common incidental radiographic finding but less common in a generalized form. External resorption can be classified into 4 categories based on clinical and histologic manifestations: external surface resorption, external inflammatory root resorption, ankylosis, and replacement resorption. In addition to these four categories, 


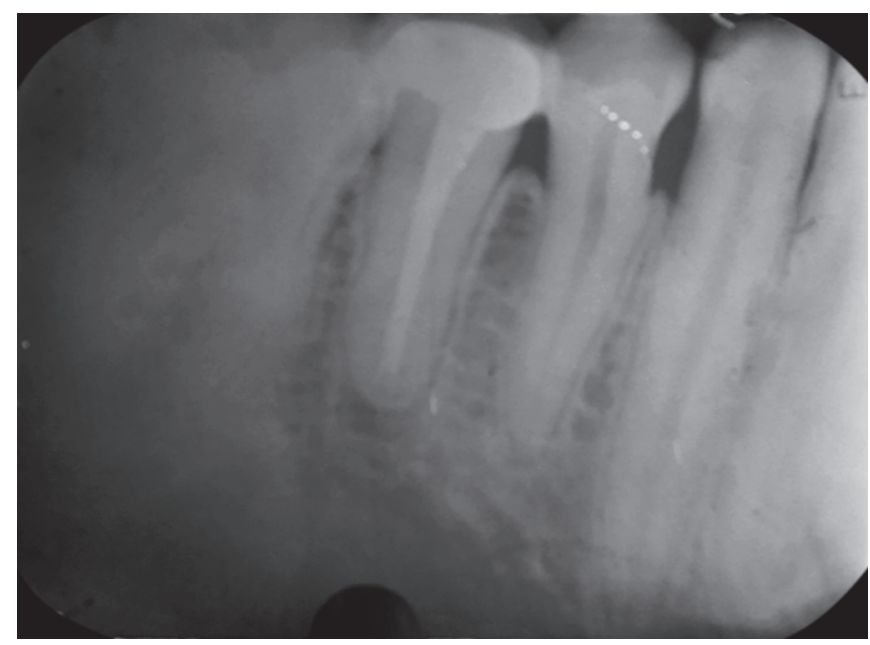

Fig. 5: Follow-up radiograph at 6 months

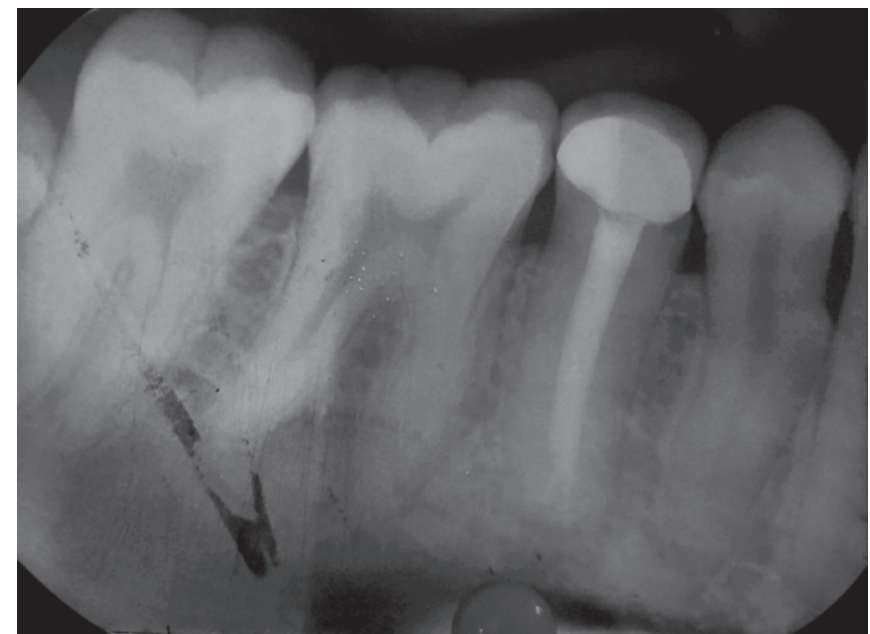

Fig. 7: Follow-up radiograph at 2 years

external resorption can also be classified based on their location in relation to the tooth: cervix, body, or apex of tooth. ${ }^{6}$

Based on etiology, different management strategies are planned for the treatment of external root resorption. The primary treatment plan for inflammatory root resorption is removal or reduction in the source of infection in the root canal. ${ }^{7}$ Andreasen suggested the best management of external root resorption by nonsurgical root canal therapy combined with a calcium hydroxide dressing to arrest root resorption. ${ }^{8}$ Intracanal medicament may help to eliminate bacteria present in the root canal, and the bacteria are best destroyed by using $\mathrm{Ca}(\mathrm{OH})_{2}$ as intracanal medicament. The main purpose of calcium hydroxide dressing as intracanal medicament is to provide disinfection, control infection, inhibit resorption, and maintain the root canal at high $\mathrm{pH}$ value of 12 . However, disadvantage of using $\mathrm{Ca}(\mathrm{OH})_{2}$ for long-term intracanal medicament ( $>30$ days) is weakening of root structure in immature teeth, which may lead to cervical root fracture. ${ }^{9}$

Untreated root resorption may lead to perforation of root which is followed by tooth fracture or progressive apical resorption that

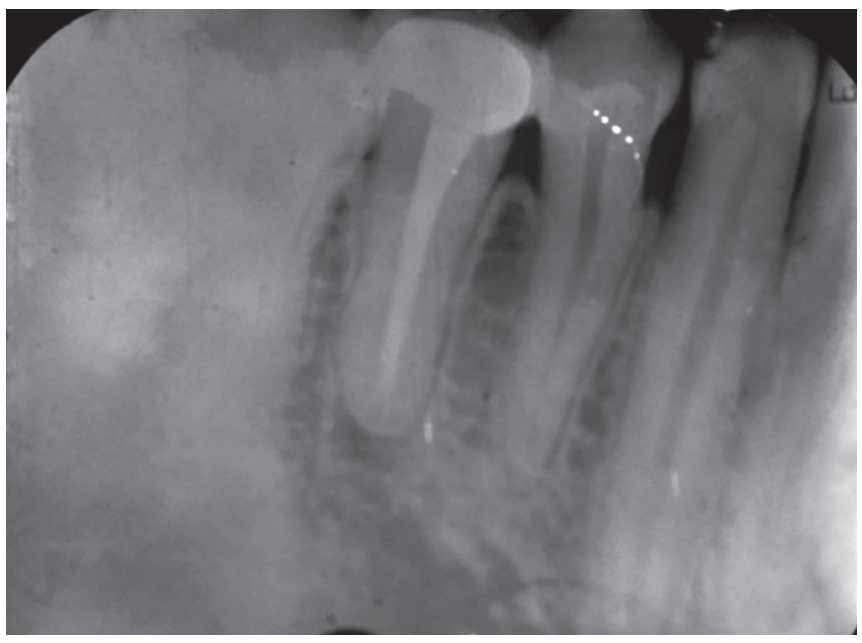

Fig. 6: Follow-up radiograph at 1 year

may lead to loss of appropriate crown-root ratio. In cases of large defects, surgical correction and filling of the communication with proper restorative material are suggested.

Dentin lost through resorption cannot be replaced by new dentin. By arresting the resorption process, healing can occur which can be replaced either with a layer of new cementum or bone and by establishment of new PDL. MTA can often used as repair material of root structure due to its superior sealing ability, biocompatibility, and fibroblastic stimulation.

\section{Conclusion}

Tooth resorption is a pathologic condition that might go unnoticed over many years, as most cases of resorption are asymptomatic in nature. For successful outcome, early diagnosis, removal of the cause, and proper treatment options in the interest of the tooth are mandatory for treatment outcome to prevent overweaking of the remaining tooth structure.

\section{References}

1. Imfeld T. Dental erosion: definition, classification and links. Eur J Oral Sci 1996;104(2):151-155. DOI: 10.1111/j.1600-0722.1996. tb00063.x.

2. Fuss Z, Tsesis I, Lin S. Root resorption - diagnosis, classification and treatment choices based on stimulation factors. Dent Traumatol 2003;19(4):175-182. DOI: 10.1034/j.1600-9657.2003.00192.x.

3. Ne RF, Witherspoon DE, Gutmann JL. Tooth resorption. Quintessence Int Pubmed 1999;30(1):9-25.

4. Hargreaves KM, Cohen S. Cohen's Pathways of the Pulp. 10th ed., Mosby Elsevier; 2011. 639-647.

5. Bellamy C, Malkhassian G. Root Resorption: An Overview and Case Report Of Endodontic Management Oral Health. 2015.

6. Gunraj MN. Dental root resorption. Oral Surg Oral Med Oral Pathol Oral Radiol Endod 1999;88(6):647-654. DOI: 10.1016/s10792104(99)70002-8.

7. Bakland LK. Root resorption. Dent Clin North Am 1992;36(2):491-507.

8. Andreasen JOAF. Textbook and Color Atlas of Traumatic Injuries to the Teeth. St. Louis: Mosby; 1994.

9. Ingle B. Baumgartner: Pathologic Tooth Resorption. Ingle's Endodontics. 6th ed., BC Decker Inc; 2008. p. 1358. 